\title{
Understanding the assembly of Kepler's tightly-packed planetary systems
}

\author{
Thomas O. Hands, Richard D. Alexander and Walter Dehnen \\ Department of Physics \& Astronomy, \\ University of Leicester, University Road, \\ Leicester, LE1 7RH, UK \\ email: tom.hands@le.ac.uk
}

\begin{abstract}
The Kepler mission has recently discovered a number of exoplanetary systems, such as Kepler 11, in which ensembles of several planets are found in very closely packed orbits. These systems present a challenge for traditional formation and migration scenarios. We present a dynamical study of the evolution of these systems using an $N$-body approach, incorporating both smooth and stochastic migration forces and a variety of initial conditions, in order to assess the feasibility of assembling such systems via traditional, disc-driven migration.
\end{abstract}

Keywords. planets and satellites: individual (Kepler-11; Kepler-32; Kepler-80), planets and satellites: dynamical evolution and stability, planets and satellites: formation

\section{Motivation}

An intriguing result of the Kepler mission is the relative abundance of a new class of closely-packed planetary systems. These systems contain 5 or 6 planets, each separated from its neighbours by fractions of an AU. The prototype for this class of systems is Kepler-11 (Lissauer et al. 2011), a 6-planet system in which the 5 innermost planets all orbit at smaller radii than Mercury. All of the planets appear to have low $(<0.05)$ eccentricities (Lissauer et al. 2013). Kepler-32 (Swift et al. 2013) and Kepler-80 (Ragozzine \& Kepler Team 2012) each contain 5 planets and are even more tightly-packed than Kepler-11. Kepler-11 displays no obvious resonant behaviour, but there are possible 3 and 4 body interlocking mean-motion resonances in Kepler-32 and -80 respectively.

It is theoretically challenging to explain how such systems might form and remain dynamically cold. If the planets underwent convergent migration, one would expect that the vast majority of adjacent pairs would be trapped in mean-motion resonances with high forced eccentricities, which is not the case. This has led several authors to consider in situ formation models (e.g., Hansen \& Murray 2013), while others have considered groups of embryos colliding and growing as they migrate inwards (e.g., Terquem \& Papaloizou 2007). We investigate the possibility of assembling these systems from planets formed further out in a disc, which then migrate to their observed locations. A full account of the method and results can be found in Hands, Alexander \& Dehnen (2014).

\section{Method}

We follow the evolution of these systems using an N-body integrator, allowing us to explore a wider parameter space than would be possible with hydrodynamical simulations. We use parametrized forces to migrate the planets and damp their eccentricities, with the time-scale of both being proportional to the inverse of the planetary mass analogous to Type I migration. We follow the method of Rein \& Papaloizou (2009), adding 

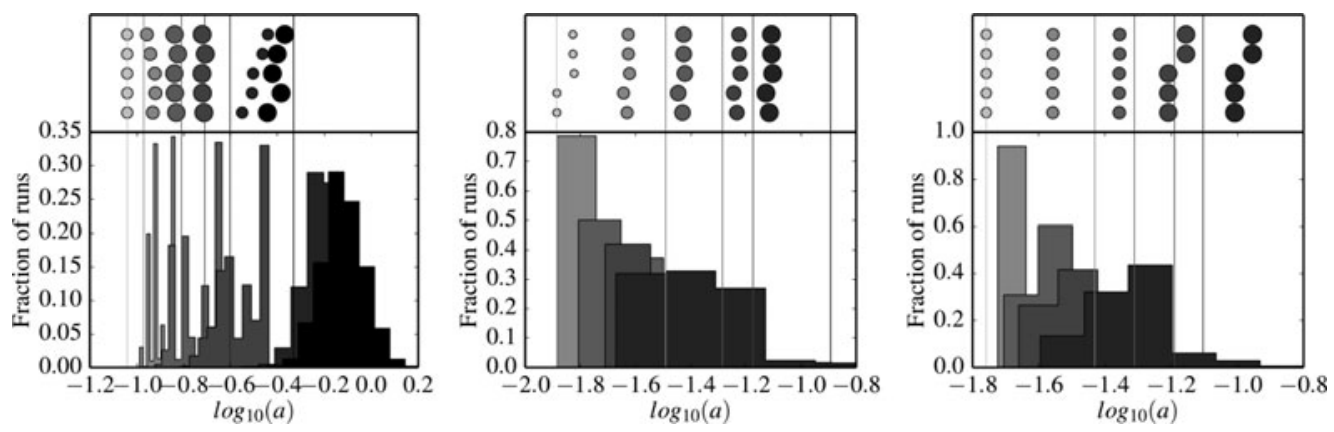

Figure 1. Histograms showing distribution of each planet in each system across all successful runs. Each shade represents a specific planet, with darker shades representing planets that are further from the star. Solid lines show the actual positions of the planets in each system, while the circles plotted above show planetary positions in some representative "best-fit" models, fitted by semi-major axis. Left to right: Kepler-11, Kepler-32, Kepler-80. Note that there is no histogram for the innermost planet in each system since this is always halted in the same place.

a stochastic component to the forces acting on the planets to simulate the effect of disc turbulence. The strength of the forces can be controlled via free parameters, and we perform 10,000 simulations with each system in order to sample the vast 3-D parameter space. We begin each simulation with random initial orbital separations and phases, and consider a simulation successful if the innermost planet reaches its observed orbital radius with the other planets in their observed order and with no collisions or ejections.

\section{Results \& Discussion}

Figure 1 shows that systems analogous to Kepler-11, -32 and -80 are easily reproduced by our model. We find a preference for weaker turbulence and short eccentricity damping time-scales, with no particular preference in migration time-scale across the range of $10^{3.5}-10^{6}$ yr that we explore. The lack of mean-motion resonances is difficult to reproduce.

This proof-of-concept model shows that forming tightly-packed planetary systems via simultaneous, disc-driven migration of fully-formed planets is possible for sensible disc models. Further work is required to understand why Type I migration should stop at the current locations of the planets, and the paucity of resonances in the observed systems.

\section{References}

Hands, T. O., Alexander, R. D., \& Dehnen, W. 2014, MNRAS, in press, arXiv:1409.0532

Lissauer, J. J., Fabrycky, D. C., Ford, E. B., Borucki, W. J., Fressin, F., Marcy, G. W., Orosz, J. A., Rowe, J. F., Torres, G., Welsh, W. F., Batalha, N. M., Bryson, S. T., Buchhave, L. A., Caldwell, D. A., Carter, J. A., Charbonneau, D., Christiansen, J. L., Cochran, W. D., Desert, J.-M., Dunham, E. W., Fanelli, M. N., Fortney, J. J., Gautier, T. N., III, Geary, J. C., Gilliland, R. L., Haas, M. R., Hall, J. R., Holman, M. J., Koch, D. G., Latham, D. W., Lopez, E., McCauliff, S., Miller, N., Morehead, R. C., Quintana, E. V., Ragozzine, D., Sasselov, D., Short, D. R., \& Steffen, J. H. 2011, Nature, 470, 53

Rein, H. \& Papaloizou, J. C. B. 2009, A\&A, 497, 595

Lissauer, J. J., Jontof-Hutter, D., Rowe, J. F., Fabrycky, D. C., Lopez, E. D., Agol, E., Marcy, G. W., Deck, K. M., Fischer, D. A., Fortney, J. J., Howell, S. B., Isaacson, H., Jenkins, J. M., Kolbl, R., Sasselov, D., Short, D. R., \& Welsh, W. F. 2013, ApJ, 770, 15 
Swift, J. J., Johnson, J. A., Morton, T. D., Crepp, J. R., Montet, B. T., Fabrycky, D. C., \& Muirhead, P. S. 2013, ApJ, 764, 14

Ragozzine, D. \& Kepler Team 2012, AAS/Division for Planetary Sciences Meeting Abstracts, 44, \# 200.04

Terquem, C. \& Papaloizou, J. C. B. 2007, ApJ, 654, 1110

Hansen, B. M. S. \& Murray, N. 2013, ApJ, 775, 17 